\title{
A ruthenium oxide thermometer for dilution refrigerators operating down to $5 \mathrm{mK}$
}

\author{
S.A. Myers, ${ }^{1}$ H. Li ${ }^{1}{ }^{1}$ and G.A. Csáthy ${ }^{1}$ \\ ${ }^{1}$ Department of Physics and Astronomy, Purdue University, West Lafayette, IN 47907
}

(Dated: October 11, 2021)

\begin{abstract}
At the lowest temperatures achieved in dilution refrigerators, ruthenium oxide resistance thermometers often saturate and therefore lose their sensitivity. In an effort to extend the range of such temperature sensors, we built a thermometer which maintains sensitivity to $5 \mathrm{mK}$. A key component of this thermometer is an in situ radio frequency filter which is based on a modern rf absorption material. We show that the use of such a filter is only effective when it is encased in the same rf-tight enclosure as the ruthenium oxide sensor. Our design delivers an attenuation level that is necessary to mitigate the effects of parasitic heating of a fraction of $\mathrm{pW}$ present in our circuit. Furthermore, we show that the likely origin of this parasitic heating is the black body radiation present within the experimental space of the refrigerator. We found that the equilibration time of the thermometer increases rapidly as the temperature is lowered; below $5 \mathrm{mK}$ this thermometer becomes impractical because of the prohibitively long equilibration times.
\end{abstract}

\section{INTRODUCTION}

Dilution refrigerators are widely used in the study of condensed matter, nanoelectronics, and quantum technologies. Modern instruments often cool to temperatures close to $5 \mathrm{mK}$. There is thus a need to routinely measure such low temperatures. Among the large variety of thermometers available for dilution refrigerators [1, thick film ruthenium oxide resistors $\left(\mathrm{RuO}_{2}\right)$ emerged as the most commonly used ones [2-12. $\mathrm{RuO}_{2}$ resistors are available as chips from electronics components vendors and from companies specializing in low temperature thermometry. Advantages of $\mathrm{RuO}_{2}$ thermometers include wide availability, ease of use, small size, excellent stability to thermal cycling, and moderate magnetoresistance.

Resistive $\mathrm{RuO}_{2}$ thermometers, however, are not without their own limitations. In many cases they become unusable typically below about $20 \mathrm{mK}[1-6$. Indeed, at these temperatures the resistance versus temperature calibration curves of $\mathrm{RuO}_{2}$ thermometers often saturate and these thermometers lose sensitivity $[1] 6$. Furthermore, below $20 \mathrm{mK}$ the resistance of $\mathrm{RuO}_{2}$ thermometers is often not reproducible and may exhibit unexplained jumps in the resistance [6]. Such a behavior was attributed to a combination of two factors: the gradual loss of thermal contact of electrons with the phonon bath as the temperature is lowered combined with parasitic radio frequency (rf) heating of electrons in the $\mathrm{RuO}_{2}$ chip [5]. Therefore below about $20 \mathrm{mK}$ one may not rely on these thermometers, and the calibration of these thermometers at the lowest temperatures will likely be off when moved to a different dilution refrigerator. The literature has two examples of $\mathrm{RuO}_{2}$ based resistors that maintained sensitivity to $6 \mathrm{mK}[11$ and to $7.8 \mathrm{mK}[12$. These thermometers are, however, not based on commercial chips, but rather prepared from a $\mathrm{RuO}_{2}$ paste [11] or a proprietary ink [12]. However, it is not clear if any material specific properties led to this improvement. The loss of sensitivity of $\mathrm{RuO}_{2}$ based resistors near the base temperature of dilution refrigerators thus remains poorly understood, and a recipe for mitigating it did not yet emerge.

Parasitic rf heating plaguing $\mathrm{RuO}_{2}$ thermometry at low temperatures is also recognized to have detrimental effect in a variety of electronic systems. Examples are single electron systems realized with metallic and semiconductor based quantum dots [13 17, sub-millikelvin Johnson noise thermometers [18, 19, quantum Hall systems [20, 21], and superconducting and semiconductor quantum dot quantum processors 22. Parasitic heating in these systems is commonly controlled with rf filters built using various absorbant materials. Examples of such filters are the ones based on epoxy impregnated metal powders [23 27], silver epoxy [28, 29, thermocoax [30, and magnetically loaded high loss dielectrics [31. However, to our knowledge, modern rf filtering technology was not used for mitigating parasitic heating in $\mathrm{RuO}_{2}$ thermometers.

In this report we set out to fabricate a $\mathrm{RuO}_{2}$ resistance thermometer based on a commercial chip that is capable of reaching temperatures as low as $5 \mathrm{mK}$. A key component of this thermometer is an in situ silver epoxy rf filter that takes advantage of several elements of modern rf filtering technology. Direct performance comparisons are made with unshielded $\mathrm{RuO}_{2}$ resistance thermometers which are shown to saturate with temperature below $20 \mathrm{mK}$. This allows us to estimate the parasitic rf heating power picked up by our circuitry as well as determine the effective temperature of the black body radiation responsible for such heating. Lastly, we discuss the practicality of our newly designed $\mathrm{RuO}_{2}$ resistance thermometer by analyzing how the thermal equilibration time scales with temperature. 


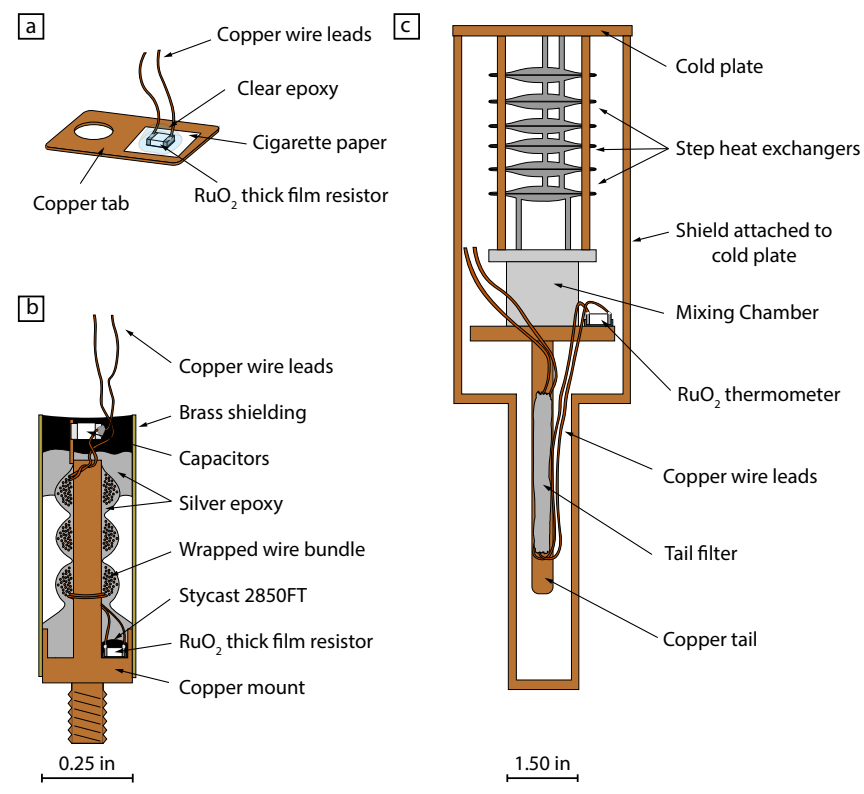

FIG. 1. Panel a: Schematic of thermometers Th-1, Th-2, and Th-3. Panel b: Schematic of Th-4. Panel c: Schematic of our measurement setup. The copper tail attached to the mixing chamber with a silver epoxy rf filter and the location of the $\mathrm{RuO}_{2}$ thermometer are clearly marked.

\section{Design of $\mathrm{RuO}_{2}$ thermometers}

In this study we use several commonly available $\mathrm{RuO}_{2}$ chips [32. Information on these chips can be found in Table.I. Thermometers Th-1, Th-2, and Th- 3 are built by attaching $\mathrm{RuO}_{2}$ chips of different size to a copper tab, and a schematic of the thermometer design is shown in Fig 17. Magnet wires are soldered onto the chip using indium, and cigarette paper is used to electrically isolate the chips from the copper tab. These chips are glued onto the copper tab face down, i.e. the conductive element and its protective insulation faces the cigarette paper and the copper tab.

A more elaborately designed thermometer Th- 4 is shown in Fig 1 1 b. Built into the rf-tight enclosure of Th-4 is a low-pass rf filter based on silver epoxy [28, 29], which we refer to as the in situ filter. We chose silver epoxy as the absorbant because of its effectiveness in absorbing rf power, its thermal conduction, and its non-magnetic

\begin{tabular}{|c||c|c|c|}
\hline \multicolumn{4}{|c|}{$\mathrm{RuO}_{2}$ Resistor Parameters } \\
\hline Thermometer & Chip size & $R(\Omega)$ & Part number \\
\hline Th-1 & 0805 & 738 & RK73H2ALTD7380F \\
Th-2 & 0805 & 300 & RK73H2ATTD3000F \\
Th-3 & 0402 & 560 & RK73H1ETTP5600F \\
Th-4 & 0402 & 560 & RK73H1ETTP5600F \\
\hline
\end{tabular}

TABLE I. Nominal parameters of the thick film $\mathrm{RuO}_{2}$ resistor chips used for the thermometers discussed. property [28, 29]. In order to enhance the effectiveness of the in situ filter, we impose the following constraints for Th-4: 1) the $\mathrm{RuO}_{2}$ chip is mounted in an rf-tight enclosure 2) the in situ rf filter is also located inside the rf-tight enclosure and it is based on a twisted pair of magnet wires encased into a silver epoxy matrix 3) wrapping of the twisted pair of wires that form the in situ filter is done in three well separated bundles and 4) two capacitors are used to channel rf waves to ground. We thus used the best practices in rf filtering that are known to lower the filter cut-off frequency, such as the use of capacitors [27, 29] and of wire bundles instead of evenly distributed windings [29. The novelty of our design is an rf-tight encapsulation of the $\mathrm{RuO}_{2}$ chip, of the in situ filter, and of the wires connecting the in situ filter and the $\mathrm{RuO}_{2}$ chip.

We describe here the components and the assembly process for Th-4. As shown in Fig 1 b, the $\mathrm{RuO}_{2}$ chip, the enclosure, and the in situ rf filter are supported by a threaded copper mount machined out of a $1 / 4$ inch diameter stock. The region where the chip is mounted has a $2 \mathrm{~mm}$ deep annular groove. A twisted pair of magnet wires of AWG 38 with heavy polyimide insulation was soldered to the $\mathrm{RuO}_{2}$ thick film resistor using indium. Afterwards, the $\mathrm{RuO}_{2}$ chip was glued face down into the groove and epoxied using Stycast 2850FT. A small piece of cigarette paper was used to prevent an electrical short to the copper mount and care was taken to minimize the amount of epoxy used. However, we made sure that the non-conductive epoxy covered all conductive parts, including the stripped magnet wire, contacts of the chip, and the indium solder. The twisted wire pair of approximately 1.5 meters in length was then wrapped around the central copper column in three roughly equally spaced bundles. The silver epoxy [33] was applied between the layers, as the wires were wrapped onto the post. We emphasize that care must be taken for the thermometer circuit not to short to ground: we relied on the Stycast epoxy to electrically insulate any exposed metallic parts and on the heavy polyimide insulation of the wires to keep the silver epoxy from shorting to the magnet wires. We then soldered each magnet wire to a $1 \mathrm{nF}$ capacitor 34 connected to ground. These capacitors are mounted near the top of the thermometer. A brass thin walled tube was slipped onto the copper mount; its bottom part was attached to the copper mount with silver epoxy, while its top part was sealed with the same type of silver epoxy. We note that when cooled, the small volume of trapped air within the brass tube will freeze. One may eliminate this open volume by filling it with silver epoxy. However, the large thermal mass of the epoxy may negatively affect thermalization times of the thermometer. Thus, we opted to use the minimum amount of silver epoxy required to produce an rf-tight enclosure. Lastly, Stycast 2850FT was used to structurally hold the capacitors and magnet wires in place. 


\section{Cryogenic setup for resistance thermometry measurement}

Our measurement setup has two rf filters: the room temperature and the tail filter. The tail filter, shown in Fig 1 , , is a silver epoxy filter located in a groove along the copper tail that is fastened to the mixing chamber plate. We use this copper tail to thermalize samples exposed to magnetic fields generated by a superconductive solenoid. Along with rf attenuation, the silver epoxy used for the tail filter provides structural support and thermally anchors the electrical leads that run along the tail. Details of these filters can be found in Ref.[28. As shown in Fig 1 , the $\mathrm{RuO}_{2}$ thermometers are mounted on the mixing chamber plate and connect to the bottom end of the tail filter. The measurement wires exiting through the top of the tail filter are heatsunk at the mixing chamber and connect to room temperature via $0.1 \mathrm{~mm}$ constantan wires provided with our dilution refrigerator by the manufacturer, Oxford Instruments. These constantan wires have a resistance near $210 \Omega$ and are heatsunk at each cooling stage of the instrument.

The four thermometers were calibrated against a carbon thermometer described in Ref. 35]. This carbon thermometer was shown to thermalize to low temperatures and was calibrated against a He-3 quartz tuning fork viscometer [28. In comparison to the carbon thermometer, we expect that our $\mathrm{RuO}_{2}$ thermometers will tolerate much reduced heating levels [12, 28. Four-wire resistance measurements were performed using a Lakeshore $370 \mathrm{AC}$ resistance bridge with a model 3716 scanner front end. As shown later, an excitation level of $100 \mathrm{pA}$ was chosen at the lowest temperatures to avoid self-heating of the $\mathrm{RuO}_{2}$ thermometers.

\section{Calibration curves for $\mathrm{RuO}_{2}$ thermometers Th-1, Th-2, and Th-3}

In Fig 2 we show the calibration of the three $\mathrm{RuO}_{2}$ thermometers affixed to copper tabs. As expected, the resistance of these thermometers increases with a decreasing temperature. As shown in the inset of Fig, larger room temperature values result in larger low temperature values. In addition, for Th- 1 and Th- 3 we observe a decrease in their sensitivity starting at $30 \mathrm{mK}$, and there is a clear saturation of the resistance below $20 \mathrm{mK}$. This behavior is similar to that reported in Refs. [2], and we associate this saturation of the calibration curve with the presence of unwanted parasitic rf heating. We recall that the circuit for measuring these thermometers contains two rf filters: one at room temperature and another silver epoxy based filter affixed to the tail. We thus find that a setup with these two rf filters does not result in an effective electronic thermalization within the $\mathrm{RuO}_{2}$ chips of the three thermometers based on copper tabs.

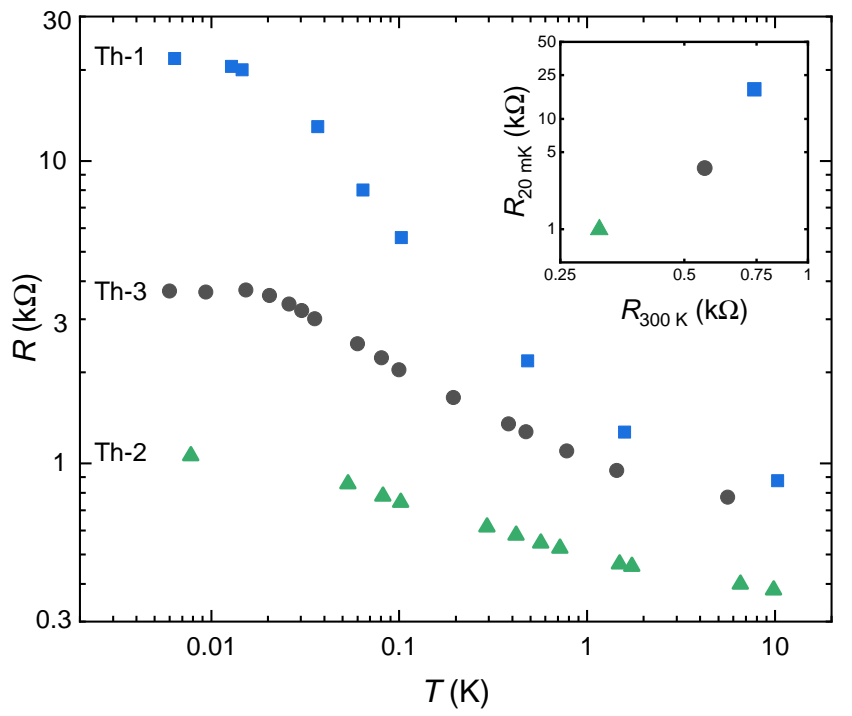

FIG. 2. Resistance calibration for various $\mathrm{RuO}_{2}$ thermometers. Thermometers Th- 1 , Th- 2 , and Th-3 have a similar design; the $\mathrm{RuO}_{2}$ chip are attached to a copper tab and cigarette paper is used to prevent shorts to ground. The inset shows the relationship of $R_{300 \mathrm{~K}}$ and $R_{20 \mathrm{mK}}$, i.e. resistances measured at room temperature and at $20 \mathrm{mK}$.

\section{Low temperature performance of Th-4 with an in situ filter}

In Fig 3 we compare the resistance versus temperature calibration curves of Th-3 and Th-4. Even though built using $\mathrm{RuO}_{2}$ chips from the same batch, the behavior of these two thermometers is quite different. Above $20 \mathrm{mK}$, both thermometers have a similar trend. Small changes in the calibration, such as the ones shown in Fig 3 , are expected when using chips taken from the same batch. However, the most striking difference between the two thermometers is in their low temperature behavior. Below about $20 \mathrm{mK}$, the resistance of Th- 4 continues to rise monotonically as the temperature is lowered. In fact the resistance as function of temperature for Th- 4 maintains the same power law $R \propto T^{-0.38}$ between $0.1 \mathrm{~K}$ and $5 \mathrm{mK}$, indicating therefore no change in the conduction mechanism over this temperature range. Thermometer Th- 4 can thus be used to measure temperature at least down to $5 \mathrm{mK}$, extending therefore the useful range for a $\mathrm{RuO}_{2}$ thermometer to the full range of dilution refrigerator temperatures. Maintaining sensitivity down to $5 \mathrm{mK}$ in Th-4 indicates the successful mitigation of the unwanted parasitic rf heat in this thermometer. We attribute the removal of the parasitic rf heat to the combination of the rf-tight enclosure and the effects of the in situ rf filter. 


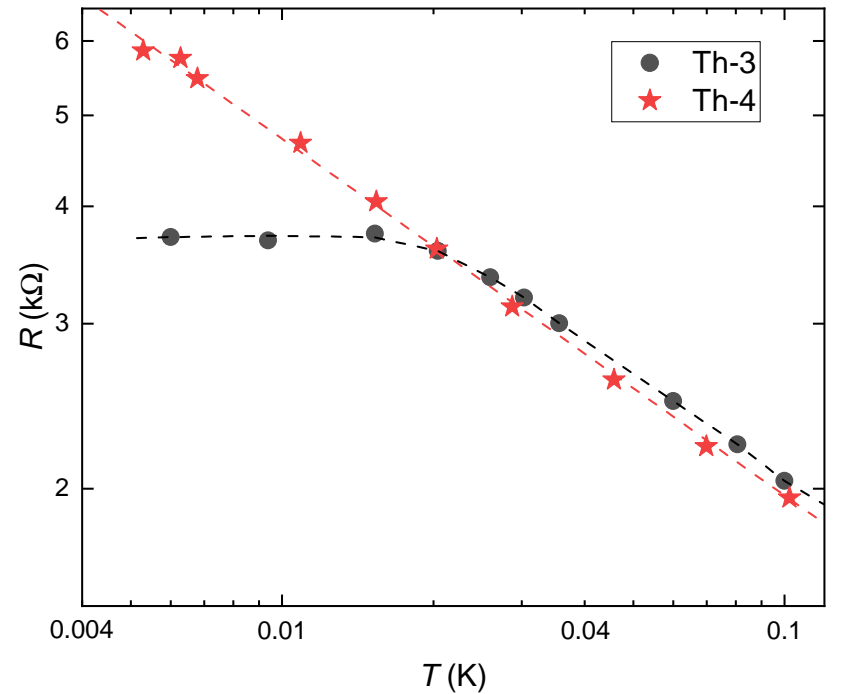

FIG. 3. A comparison of the low temperature calibration of Th- 3 and Th-4. These thermometers are built using $\mathrm{RuO}_{2}$ chips of identical size and nominal resistance, but Th- 4 has an in situ rf filter and is sealed in an rf-tight enclosure. Dashed lines are guides for the eye. The calbration curve of Th-3 saturates below about $20 \mathrm{mK}$. In stark contrast, Th- 4 maintains its sensitivity to the lowest temperatures.

\section{Self-heating analysis}

In the most general case, the power dissipated through a $\mathrm{RuO}_{2}$ chip is $P=I^{2} R+P_{\text {paras. }}$. Here $I$ is the current excitation, $I^{2} R$ the Joule heating of this excitation, and $P_{\text {paras }}$ the parasitic rf heating. However, the in situ filter used in the construction of Th-4 effectively dissipates the parasitic rf heating. Thus for Th-4 the parasitic rf heating is assumed to be negligible $P_{\text {paras }} \approx 0$. The non-saturating resistance of Th- 4 at the lowest temperatures, shown in Fig 3 supports the validity of this assumption. By performing a so called self-heating experiment, we establish the upper bound on the excitation levels which will not overheat this thermometer. For this measurement the mixing chamber was held fixed at $T=6.7 \mathrm{mK}$ and $I$ was successively increased. With an increasing excitation level, Joule heating causes the electronic temperature to rise, hence the resistance decreases. The observed drop in resistance was converted to an increase in temperature using the calibration curve shown on Fig, 3 . The resulting self-heating curve is shown in Fig 4 . We found that near the base temperature the electron temperature in the conductive channel of the $\mathrm{RuO}_{2}$ chip starts decoupling from the phonon bath temperature at around $1 \mathrm{fW}$ of heating power. This value is comparable with measured values at $8 \mathrm{mK}[12,28$. Such power levels require excitation currents of about $I=100 \mathrm{pA}$. Hence such low excitation levels were used for calibration at the low end of the temperature scale.

The self-heating measurement also allows us to char-

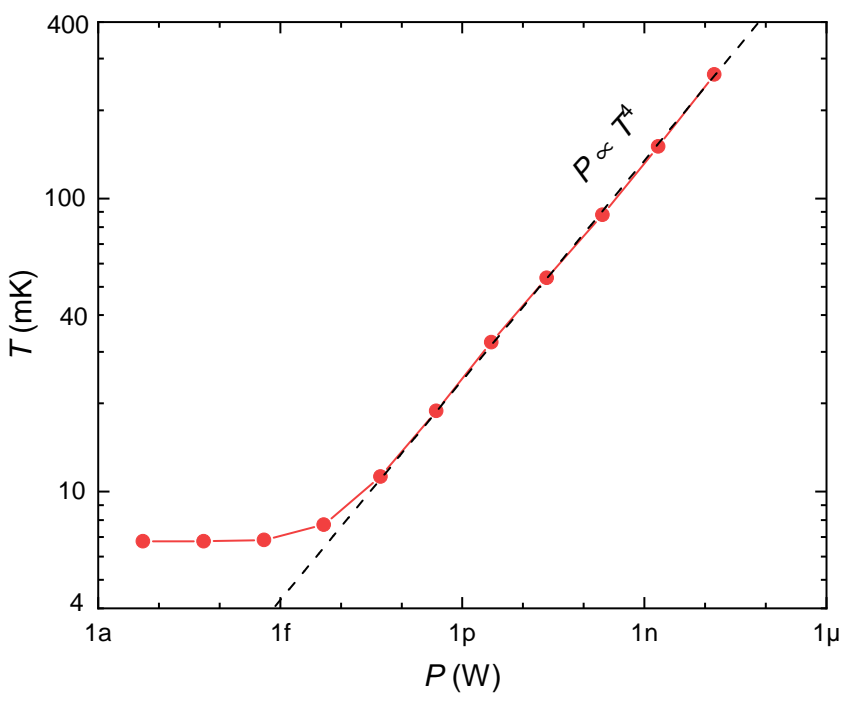

FIG. 4. Self-heating curve $T$ versus the Joule excitation power $P$ of the thermometer Th-4 built with an in situ rf filter. The mixing chamber during this measurement was held at $T=6.7 \mathrm{mK}$. The temperature reading of this thermometer starts to rise at an excitation level of about $1 \mathrm{fW}$. The dashed line is $P \propto T^{4}$.

acterize the rf environment of the thermometers. In contrast to Th-4, Th-3 lacks the in situ rf filter and therefore, is not thermalized to the base temperature due to parasitic rf heating. We recall that the resistance of Th-3 saturates near $T=20 \mathrm{mK}$. According to the self-heating data shown in Fig 4 , a Joule heating power of $0.45 \mathrm{pW}$ is needed to heat Th-4 to $20 \mathrm{mK}$ when the mixing chamber is kept cold. Thus, we surmise the same power level $P_{\text {paras }}=0.45 \mathrm{pW}$ is present in our circuit and causes the saturation of the resistance of Th-3.

Since Th- 4 thermalizes to $5 \mathrm{mK}$, its in situ filter is effective in attenuating the $0.45 \mathrm{pW}$ parasitic power to below $1 \mathrm{fW}$. We therefore estimate the attenuation level required for the in situ filter in order to effectively cool our $\mathrm{RuO}_{2}$ chip to $5 \mathrm{mK}$ to be $\approx 10 \log 0.45 \mathrm{pW} / 1 \mathrm{fW}=27 \mathrm{~dB}$, or nearly a factor of 1000 . Such a figure is reasonable since a filter built of similar elements had an attenuation exceeding this value in the $2 \mathrm{MHz}$ to $10 \mathrm{GHz}$ frequency band [29. Nonetheless, one must keep in mind that those attenuation measurements were done in a $50 \Omega$ environment. In the present case, a direct measurement of the spectral distribution of the attenuation in a $\mathrm{RuO}_{2}$ chip is not readily possible since changes with temperature of the $\mathrm{RuO}_{2}$ load resistance will cause a change in the frequency dependent attenuation. Evidence for such an effect can be found in a Johnson noise thermometry experiment in which parasitic heating led to the saturation of the electronic temperature that was dependent on the load resistance 19 . We think that in contrast to the broad band nature of microwave circuits built with coaxial lines and with $50 \Omega$ sources and loads, in our circuit 
the power is effectively coupled into Th-3 through a set of discrete frequencies. Therefore in our circuit a bolometric measurement of the power through the earlier described self-heating measurement is more meaningful.

\section{Effective temperature of black body radiation}

In order to identify the origin of the $0.45 \mathrm{pW}$ parasitic power the thermometer Th-3 experiences, it is important to recognize that rf radiation may couple to this thermometer in two distinct ways. First, rf heating may originate from outside the dilution refrigerator; rf waves from our lab may travel along the measurement wires toward the $\mathrm{RuO}_{2}$ chip. Second, rf heating may originate from inside the dilution refrigerator, as a black body radiation present in the environment of the $\mathrm{RuO}_{2}$ chip. As seen in Fig 1k, a length of about 0.3 meters of the measurement wires leaving the bottom of the tail filter and connecting to the $\mathrm{RuO}_{2}$ chip on the mixing chamber plate is not shielded and is thus exposed to the black body radiation present in this region. The most striking difference in the construction of Th- 3 and Th- 4 is that the former is directly exposed to this black body radiation, whereas the latter is not. We thus think that a major contributor to the saturation of the low temperature calibration of Th-3 is this black body radiation. Assuming that the measurement wires are fully thermalized by the heat sinks and tail filter and assuming an ideal emisittivity, we can provide a crude estimate for $\widetilde{T}$, the effective temperature of a black body radiating the $P=0.45 \mathrm{pW}$ power into our thermometer circuit. From the Stefan-Boltzmann law $P=\sigma A \widetilde{T}^{4}$ we estimate $\widetilde{T} \approx 0.4 \mathrm{~K}$. Here $A$ is the area of the exposed wires that run from the bottom of the tail filter to the thermometer. $\widetilde{T}$ is significantly larger than the $90 \mathrm{mK}$ temperature of the cold plate and the radiation shield attached to it. Black body radiation from the liquid helium bath of our wet refrigerator penetrating through openings of the cold plate may explain such an effective temperature $\widetilde{T}$. In typical instruments, such as ours, the cold plate and its shield do not form an rftight enclosure as there are through holes and openings on the cold plate near the wiring, for example. Our results highlight therefore the necessity of blocking such a radiation from entering the channel of the $\mathrm{RuO}_{2}$ chip. Moreover, the four constraints put forth in the design of Th-4 can also be used to effectively cool any electronic device to millikelvin temperatures or below.

\section{Equilibration time of Th-4}

While the improved low temperature resistive behavior of Th-4 is evident when compared against a typical $\mathrm{RuO}_{2}$ thermometer, such as Th-3, there remains another critical aspect for practicality purposes: the time required

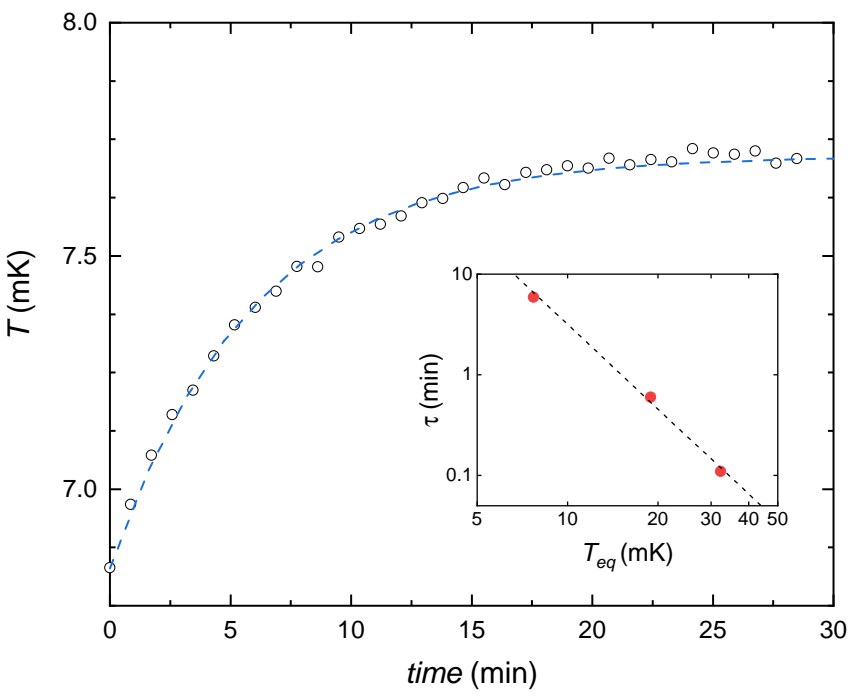

FIG. 5. Time evolution reading of Th-4 when its excitation is increased from $316 \mathrm{pA}$ to $1 \mathrm{nA}$. Here the mixing chamber temperature is held at $6.7 \mathrm{mK}$. The dashed line is an exponential fit from which the time constant $\tau$ is extracted. The inset shows the dependence of the time constant $\tau$ on the equilibration temperature $T_{e q}$ of the conduction channel of the thermometer. The dashed line is a power law fit of the time constants $\tau \propto T_{e q}^{-2.8}$.

for the thermometer to equilibrate. It is known that the thermal response of any type of resistive thermometer is strongly temperature dependent in the millikelvin temperature range. We quantify trends in the equilibration time by analyzing the transient behavior after injecting additional power into the thermometer. A representative response is shown in Fig.5. The transient behavior is exponential to a good degree. Time constants $\tau$ are extracted when the mixing chamber temperature is held at a fixed value and the excitation current is successively increased from $316 \mathrm{pA}$ to $1 \mathrm{nA}, 3.16 \mathrm{nA}$, and $10 \mathrm{nA}$. Shown in the inset of Fig 5 is the dependence $\tau$ on the equilibration temperature $T_{e q}$, the temperature at which transient behavior subsides. We find that for temperatures below $40 \mathrm{mK}, \tau$ increases rapidly according to the power law $\tau \propto T_{e q}^{-2.8}$. Thus, while Th-4 remains sensitive at temperatures below $20 \mathrm{mK}$, the time required for the thermometer to stabilize becomes increasingly longer with lower temperatures. Even though Th-4 may be used down to $5 \mathrm{mK}$, we expect that its response time at significantly lower temperatures becomes prohibitively long to a point at which $\mathrm{RuO}_{2}$ resistive thermometry becomes impractical.

\section{Conclusions}

To summarize, we designed a $\mathrm{RuO}_{2}$ thick film resistor based thermometer which follows the phonon bath tem- 
perature down to $5 \mathrm{mK}$. We achieved such a thermalization by attenuating parasitic radio frequency waves traveling along the measurement wires using an in situ silver epoxy filter and by using an rf-tight enclosure of the $\mathrm{RuO}_{2}$ chip, the in situ filter, and wiring connecting these elements. Furthermore, we think that the parasitic radio frequency heating originated not from the room temperature parts of our circuit but instead from the black body radiation present at the location of the thermometer. Thus, our findings highlight that $\mathrm{rf}$ shielding within the experimental space of both the electrical components and measurement wires is likely necessary for many devices to reach low millikelvin temperatures and below. From selfheating curves we found that the thermometer's in situ rf filter effectively dissipates the $0.45 \mathrm{pW}$ of radio frequency heating power. Lastly, analysis of the self-heating transient behavior shows an undesirable rapid increase in the equilibration times with decreasing temperature. Thus, the striking improvement in sensitivity comes at a cost of increased thermal response times, seriously limiting the use of the thermometer below $5 \mathrm{mK}$.

\section{Acknowledgments}

This work was supported by the US DOE, Office of Basic Energy Sciences under the award DE-SC0006671.

[1] C. Enss and S. Hunklinger, Low-Temperature Physics, Springer, Berlin (2005).

[2] H. DOI, Y. Narahara, Y. Oda, and H. Nagano, New resistance thermometer with small magnetic field dependence for low temperature measurements, Proceedings of the 17th International Conference on Low Temperature Physics LT-17, 405 (1984).

[3] Q. Li, C. Watson, R. Goodrich, D. Haase, and H. Lukefahr, Thick film chip resistors for use as low temperature thermometers, Cryogenics 2, 467 (1986). https://doi.org/10.1016/0011-2275(86)90095-0

[4] W. Schoepe, Conduction mechanism in granular $\mathrm{RuO}_{2}-$ based thick-film resistors, Physica B 165/166, 299 (1990). 10.1016/s0921-4526(90)80999-y

[5] M. Watanabe, M. Morishita, and Y. Ootuka, Magnetoresistance of $\mathrm{RuO}_{2}$-based resistance thermometers below $0.3 \mathrm{~K}$, Cryogenics 41143 (2001). https://doi.org/10.1016/s0011-2275(01)00066-2

[6] P. Jorba, Calibration of $\mathrm{RuO}_{2}$ commercially available resistors for temperature sensing at dilution refirerator temperatures, TP IV Report supervised by H. Ronnow and J. Piatek, l'Ecole Polytechnique Fédérale de Lausanne (2013).

[7] A. Briggs, Characterization of some chip resistors at low temperatures, Cryogenics 31, 932 (1991). https://doi.org/10.1016/0011-2275(91)90216-j

[8] K. Uhlig, Magnetoresistance of thick-film chip resistors at millikelvin temperatures, Cryogenics 35, 525 (1995). https://doi.org/10.1016/0011-2275(95)98220-u
[9] R. Goodrich, D. Hall, E. Palm, and T. Murphy, Magnetoresistance below $1 K$ and temperature cycling of ruthenium oxide-bismuth ruthenate cryogenic thermometers, Cryogenics 38, 221 (1998). https://doi.org/10.1016/s0011-2275(97)00100-8

[10] C.J. Yeager, S.S. Courts, and W.E. Davenport, Thermal resistance of cryogenic thermometers at ultra-low temperatures, AIP Conference Proceedings 613, 1644 (2002). https://doi.org/10.1063/1.1472201

[11] R. Willekers, F. Mathu, H. Meijer, and H. Postma, Thick film thermometers with predictable $R-T$ characteristics and very low magnetoresistance below $1 \mathrm{~K}$, Cryogenics 30, 351 (1990). https://doi.org/10.1016/00112275(90)90315-4

[12] S.S. Courts, and J.K. Krause, A commercial ruthenium oxide thermometer for use to 20 millikelvin, AIP Conference Proceedings 985, 947 (2008). https://doi.org/10.1063/1.2908694

[13] D. Vion, P.F. Orfila, P. Joyez, D. Esteve, and M.H. Devoret, Miniature electrical filters for singleelectron devices, J. Appl. Phys. 77, 2519 (1995). https://doi.org/10.1063/1.358781

[14] L. Casparis, M. Meschke, D. Maradan, A.C. Clark, C.P. Scheller, K.K. Schwarzwälder, J.P. Pekola, and D.M. Zumbühl, Metallic Coulomb blockade thermometry down to $10 \mathrm{mK}$ and below, Rev. Sci. Instrum. 83, 083903 (2012). https://doi.org/10.1063/1.4744944

[15] D. Maradan, L. Casparis, T.-M. Liu, D.E.F. Biesinger, C.P. Scheller, D.M. Zumbühl, J.D. Zimmerman, and A.C. Gossard, GaAs quantum dot thermometry using direct transport and charge sensing, J. Low Temp. Phys. 175, 784 (2014). https://doi.org/10.1007/s10909-014-1169-6

[16] M. Sarsby, N. Yurttagül, and A. Geresdi, $500 \mathrm{mi}$ crokelvin nanoelectronics, Nat. Commun. 11, 1492 (2020). https://doi.org/10.1038/s41467-020-15201-3

[17] A.T. Jones, C.P. Scheller, J.R. Prance, Y.B. Kalyoncu, D.M. Zumbühl, and R.P. Haley, Progress in cooling nanoelectronic devices to ultra-low temperatures, J. Low Temp. Phys. 201, 772 (2020). https://doi.org/10.1007/s10909-020-02472-9

[18] C.P. Lusher, J. Li, V.A. Maidanov, M.E. Digby, H. Dyball, A. Casey, J. Nyéki, V.V. Dmitriev, B.P. Cowan, and J. Saunders, Current sensing noise thermometry using a low $T_{c} D C S Q U I D$ preamplifier, Meas. Sci. Technol. 12, 1 (2001). https://doi.org/10.1088/0957-0233/12/1/301

[19] A. Casey, F. Arnold, L.V. Levitin, C.P. Lusher, J. Nyéki, J. Saunders, A. Shibahara, H. van der Vliet, B. Yager, D. Drung, Th. Schurig, G. Batey, M.N. Cuthbert, and A.J. Matthews, Current sensing noise thermometry: A fast practical solution to low temperature measurements, J. Low Temp. Phys. 175, 764 (2014). https://doi.org/10.1007/s10909-014-1147-z

[20] W. Pan, J.-S. Xia, V. Shvarts, D.E. Adams, H.L. Stormer, D.C. Tsui, L.N. Pfeiffer, K.W. Baldwin, and K.W. West, Exact quantization of the even-denominator fractional quantum Hall state at $\nu=5 / 2$ Landau level filling factor, Phys. Rev. Lett. 83, 3530 (1999). https://doi.org/10.1103/PhysRevLett.83.3530

[21] G.A. Csáthy, chapter 5 in Fractional Quantum Hall Effects: New Developments, edited by B.I. Halerin and J.K. Jain, (World Scientific, 2020).

[22] N.P. de Leon, K.M. Itoh, D. Kim, K.K. Mehta, T.E. Northup, H. Paik, B.S. Palmer, N. Samarth, S. Sangtawesin, and D.W. Steuerman, Mate- 
rials challenges and opportunities for quantum computing hardware, Science 372, 6539 (2021). https://doi.org/10.1126/science.abb2823

[23] J.M. Martinis, M.H. Devoret, and J. Clarke, Experimental tests for the quantum behaviour of a macroscopic degree of freedom: The phase difference across a Josephson junction, Phys. Rev. B 35, 4682 (1987). https://doi.org/10.1103/PhysRevB.35.4682

[24] A. Fukushima, A. Sato, A. Iwasa, Y. Nakamura, T. Komatsuzaki, and Y. Sakamoto. Attenuation of microwave filters for single-electron tunneling experiments IEEE Trans. Instrum. Meas. 46, 289 (1997). https://doi.org/10.1109/19.571834

[25] K. Bladh, D. Gunnarsson, E. Hürfeld, S. Devi, C. Kristoffersson, B. Smålander, S. Pehrson, T. Claeson, P. Delsing, and M. Taslakov, Comparison of cryogenic filters for use in single electronics experiments, Rev. Sci. Instrum. 74, 1323 (2003). https://doi.org/10.1063/1.1540721

[26] F.P. Milliken, J.R. Rozen, G.A. Keefe, and R.H. Koch, $50 \Omega$ characteristic impedance low-pass metal powder filters, Rev. Sci. Instrum 78, 024701 (2007). https://doi.org/10.1063/1.2431770

[27] A. Lukashenko, and A.V. Ustinov, Improved powder filters for qubit measurements, Rev. Sci. Instrum. 79, 014701 (2008). https://doi.org/10.1063/1.2827515
[28] N. Samkharadze, A. Kumar, M.J. Manfra, L.N. Pfeiffer, K.W. West, and G.A. Csáthy, Integrated electronic transport and thermometry at milliKelvin temperatures and in strong magnetic fields Rev. Sci. Instrum. 82, 053902 (2011). https://doi.org/10.1063/1.3586766

[29] C.P. Scheller, S. Heizmann, K. Bedner, D. Giss, M. Meschke, D.M. Zumbühl, J.D. Zimmerman, and A.C. Gossard, Silver-epoxy microwave filters and thermalizers for millikelvin experiments, App. Phys. Lett. 104, 21106 (2014). https://doi.org/10.1063/1.4880099

[30] A.B. Zorin, The thermocoax cable as the microwave frequency filter for single electron circuits, Rev. Sci. Instrum. 66, 4296 (1995). https://doi.org/10.1063/1.1145385

[31] D.F. Santavicca and D.E. Prober, Impedance-matched low-pass stripline filters, Meas. Sci. Technol. 19, 087001 (2008). https://doi.org/10.1088/0957-0233/19/8/087001

[32] RK73H series from KOA Speer Electronics Inc., purchased from Digikey or Mouser

[33] MG Chemicals, Adhesive 8331D-A/B

[34] GRM1885C1H102JA01J from Murata

[35] N. Samkharadze, A. Kumar, and G. A. Csáthy, A new type of carbon resistance thermometer with excellent thermal contact at millikelvin temperatures, J. Low Temp. Phys. 160, 246 (2010). https://doi.org/10.1007/s10909010-0192-5 